\title{
MULTI-SESSION EXTENSION OF THE JOINT-DETECTION FRAMEWORK IN FMRI
}

\author{
Solveig Badillo ${ }^{1,2}$, Thomas Vincent ${ }^{1,3}$ and Philippe Ciuciu ${ }^{1,2}$ \\ ${ }^{(1)}$ CEA/DSV/I²BM NeuroSpin center, Bât. 145, F-91191 Gif-sur-Yvette, France \\ ${ }^{(2)}$ Parietal Project Team, INRIA Saclay Ile-de-France, 91893 Orsay, France. \\ ${ }^{3}$ INRIA, MISTIS, Grenoble University, LJK, Grenoble, France
}

\begin{abstract}
Modern cognitive experiments in functional Magnetic Resonance Imaging (fMRI) involve the repetition of the same paradigm over several short sessions (or runs) since long fMRI acquisitions usually place the subject in an uncomfortable situation and generate motion artifacts. Also, shorter sessions enable to better control the subject's cognitive state and guarantee his attention during task. The Joint Detection-Estimation (JDE) framework which aims at detecting evoked activity and estimating hemodynamic responses jointly, has been developed so far to treat each session independently and then build average contrasts of interest as already done in other packages (SPM, FSL). Here, we extend JDE to the multi-session context by proposing a new hierarchical Bayesian modeling including an additional layer to describe the link between session-specific and mean evoked activity. In contrast, the HRF shape to be estimated in each region is assumed constant across sessions. Our results on simulated and real multi-session datasets show that the proposed extension outperforms its ancestor both in terms of activated areas and HRF recovery.
\end{abstract}

Index Terms - Brain activity, hemodynamics, JDE, fMRI, Bayesian inference, multisession

\section{INTRODUCTION}

In the context of fMRI data analyses, the present paper is a contribution to encoding methods. In such studies, two main concerns arise at the subject-level analysis: (i) a precise localization of evoked brain activity elicited by sensorimotor or cognitive tasks, and (ii) a robust estimation of the underlying hemodynamic response associated with these activations. Since these two steps are inherently linked, the Joint Detection-Estimation (JDE) approach [1,2], has been proposed to face these issues in a coordinated formalism. This approach performs a multivariate inference for both detection and estimation. It makes use of a regional bilinear generative model of the BOLD response and constrains parameter estimation by physiological priors using temporal and spatial information in a Markovian model. The efficiency and usefulness of this approach has been validated at the group level in [3] considering single-session datasets.

So far, the JDE framework has been developed for single session (SS-JDE) fMRI datasets. However, neuroscientists most often optimize their paradigm so as to make each session short enough in order to limit subject's movements and maintain volonteer's attention/cooperation at a high level. As a consequence, the experiment can be repeated along four to eight sessions. A basic idea to process a multi-session dataset using the standard JDE methodology consists of iterating over each session independently and then averaging activation maps and Hemodynamic Response Function (HRF) estimates afterwards. Hemodynamic fluctuations have been studied in depth since the last decade [4], revealing that between-session variability of the HRF shape can be neglected in comparison with the betweenregion and between-subject variance components. In [8] for example, although the response has been found variable accross sessions for voxels not activated in all sessions, most activated voxels have a quite stable hemodynamic response. Hence, it makes sense to consider a fixed effect HRF model across sessions by pulling all fMRI time series acquired over the different runs and searching for a single HRF shape. Also, complementary studies have highlighted the potentially strong between-session variability of activation levels in a given subject $[5,6]$ suggesting the importance of modeling this variance component through for instance a random effect model across sessions.

The aim of this paper is to propose a multi-session extension of the JDE approach [1] that embeds all the above mentioned features in order to recover robust region-based HRF estimates and reliable activation maps irrespective of the noise level fluctuations that arise across sessions.

The rest of the paper is organized as follows. Section 2 summarizes the BOLD signal model involved in the standard JDE framework before considering its extension to the multi-session case (named MS-JDE model). The bayesian inference carried out to estimate the variables is then briefly summarized in Section 3. Section 4 is devoted to results on simulated and real multi-session datasets. In particular, we compare our method with the standard single-session approach in terms of detection activation performance and HRF estimation robustness and show typical actual situations in which the proposed multi-session scheme outperforms its ancestor. Conclusions are drawn in Section 5.

\section{MULTI-SESSION JDE) MODELING}

\subsection{Single-session Joint-Detection Estimation framework}

The JDE model relies on a prior parcellation of fMRI data into $\left(\mathcal{P}_{\gamma}\right)_{\gamma=1: \Gamma}$ functionally homogeneous parcels, deriving from a spatially constrained hierarchical clustering of functional features extracted via a classical GLM analysis [7] (usually, $\Gamma$ is close to 500).

At voxel $j \in \mathcal{P}_{\gamma}$ with $\left|\mathcal{P}_{\gamma}\right|=J_{\gamma}$, the fMRI time series $\boldsymbol{y}_{j}$ is measured at times $\left(t_{n}\right)_{n=1: N}$ where $t_{n}=n \mathrm{TR}, N$ being the number of scans and TR the time of repetition. In a single-session context [1], the BOLD signal is modelled in a given parcel $\mathcal{P}_{\gamma}$ as follows:

$$
\forall j \in \mathcal{P}_{\gamma}, \quad \boldsymbol{y}_{j}=\sum_{m=1}^{M} \boldsymbol{a}_{j}^{m} \boldsymbol{X}^{m} \boldsymbol{h}_{\gamma}+\boldsymbol{P} \boldsymbol{\ell}_{j}+\boldsymbol{b}_{j} .
$$


The unknown HRF shape $\boldsymbol{h}_{\gamma}$ is constant within parcel $\mathcal{P}_{\gamma}$ but the NRLs $a_{j}^{m}$, which model the magnitude of activation, may vary in space and across stimulus types or experimental conditions. We denote the NRLs as $\boldsymbol{A}=\left\{\boldsymbol{a}^{m}, m=1: M\right\}$ with $\boldsymbol{a}^{m}=\left\{a_{j}^{m}, j \in \mathcal{P}\right\}, a_{j}^{m}$ being the effect size at voxel $j$ for condition $m$. Each NRL is assumed to be in one of $I$ groups specified by activation class assignment variables $\boldsymbol{Q}=\left\{\boldsymbol{q}^{m}, m=1: M\right\}$ where $\boldsymbol{q}^{m}=\left\{q_{j}^{m}, j \in \mathcal{P}\right\}$ and $q_{j}^{m}$ represents the activation class at voxel $j$ for condition $m$. We consider here two classes, for activated $(i=2)$ and non-activated $(i=1)$ voxels. $\boldsymbol{X}^{m}$ denotes the $N \times(D+1)$ binary matrix $\boldsymbol{X}_{m}=\left\{x_{m}^{n-d \Delta t}, n=1 \ldots N, d=\right.$ $0 \ldots D\}$ that provides information on the stimulus occurrences for the $m$ th experimental condition. $\Delta t<T R$ being the sampling period of the unknown HRFs. Vector $\boldsymbol{h}_{\gamma} \in \mathbb{R}^{D+1}$. Finally, matrix $\boldsymbol{P}=\left[\boldsymbol{p}_{1}, \ldots, \boldsymbol{p}_{O}\right]$ of size $N \times O$ comprises the values at times $t_{n}$ of an orthonormal basis (i.e., $\boldsymbol{P}^{\mathrm{t}} \boldsymbol{P}=\boldsymbol{I}_{O}$ ) consisting of $O$ functions $\boldsymbol{p}_{o}=\left(\boldsymbol{p}_{o, t_{n}}\right)^{\mathrm{t}}$ that take a potential drift and any other nuisance effect (eg. motion parameters) into account. Vector $\boldsymbol{\ell}_{j}=\left(l_{o, j}\right)_{1 \leqslant o \leqslant O}^{\mathrm{t}}$ contains the corresponding unknown regression coefficients in $V_{j}$. For the sake of simplicity, a white noise $\boldsymbol{b}_{j} \sim \mathcal{N}\left(\mathbf{0}, \sigma_{j}^{2} \boldsymbol{I}_{N}\right)$ is considered here in all JDE formulations.

\subsection{The JDE multi-session extension}

BOLD signal modeling Model (1) is extended to account for different activation amplitudes across sessions. Let $s$ denote the session index and $\boldsymbol{A}^{s}=\left\{\boldsymbol{a}^{m, s}, m=1: M, s=1: S\right\}$ the sessiondependent NRLs with $\boldsymbol{a}^{m, s}=\left\{a_{j}^{m, s}, j \in \mathcal{P}\right\}$. In contrast, in a given parcel, the HRF shape $\boldsymbol{h}_{\gamma}$ representing the vascular component of the BOLD signal, is assumed constant over all sessions.

In addition, as the noise level may fluctuate, session-specific noise variances $\sigma_{j, s}^{2}$ are introduced. Since the physiological factors and motion may fluctuate across sessions, the counfound coefficients are also session-dependent and they are denoted by $\boldsymbol{L}^{s}=$ $\left\{\ell_{j}^{s}, j \in \mathcal{P}\right\}$. The link to the observed BOLD data is thus specified via the following BOLD signal model:

$$
\forall j \in \mathcal{P}_{\gamma}, \forall s, \quad \boldsymbol{y}_{j}^{s}=\sum_{m=1}^{M} \boldsymbol{a}_{j}^{m, s} \boldsymbol{X}^{m, s} \boldsymbol{h}_{\gamma}+\boldsymbol{P} \boldsymbol{\ell}_{j}^{s}+\boldsymbol{b}_{j}^{s},
$$

where $\boldsymbol{b}_{j}^{s} \sim \mathcal{N}\left(\mathbf{0}, \sigma_{j, s}^{2} \boldsymbol{I}_{N_{s}}\right)$ and $s=1: S$. Without loss of generality, we can assume that $\boldsymbol{X}^{m, s}=\boldsymbol{X}^{m} \forall s$, because except in selfpaced designs, most often the experimental design remains constant over sessions. In what follows, the number of scans is assumed constant over sessions: $N_{s}=N, \forall s$, again without loss of generality.

Let us denote $\mathbb{Y}=\left\{\boldsymbol{Y}^{s}, s=1: S\right\}$ with $\boldsymbol{Y}^{s}=\left\{\boldsymbol{y}_{j}^{s}, j \in \mathcal{P}\right\}$ the fMRI time-series acquired during session $s$ and $\mathbb{A}=\left\{\boldsymbol{A}^{s}\right\}, \mathbb{L}=$ $\left\{\boldsymbol{L}^{s}\right\}$ the sets of NRLs and drifts pulled over sessions. Let us also introduce $\boldsymbol{\Sigma}=\left\{\boldsymbol{\sigma}^{s}, s=1: S\right\}$ with $\boldsymbol{\sigma}^{s}=\left(\sigma_{j, s}^{2}\right)_{1 \leq j \leq J_{\gamma}}$ the noise variances for session $s$. Then, given Eq. (2), the likelihood factorizes over sessions The likelihood for session $s$ reads:

$$
\begin{aligned}
& \qquad p\left(\boldsymbol{Y}^{s} \mid \boldsymbol{A}^{s}, \boldsymbol{H}_{\gamma} ; \boldsymbol{L}^{s}, \boldsymbol{\sigma}^{s}\right) \propto \prod_{j=1}^{J_{\gamma}} \sigma_{j, s}^{-N} \exp \left(-\left(\overline{\boldsymbol{y}}_{j}^{s}\right)^{\mathrm{t}} \overline{\boldsymbol{y}}_{j}^{s} / 2 \sigma_{j, s}^{2}\right) \\
& \text { with } \overline{\boldsymbol{y}}_{j}^{s}=\boldsymbol{y}_{j}^{s}-\boldsymbol{P}^{s} \boldsymbol{\ell}_{j}^{s}-\sum_{m=1}^{M} a_{j}^{m, s} \boldsymbol{X}_{m} .
\end{aligned}
$$

New hierarchical model and NRL priors To model the link between session-dependent and mean NRLs, an additional layer in the hierarchical JDE prior model [1] is introduced. Let $\bar{A}=$ $\left\{\overline{\boldsymbol{a}}^{m}, m=1: M\right\}$ be the average activity across sessions with $\overline{\boldsymbol{a}}^{m}=\left\{\bar{a}_{j}^{m}, j=1: J_{\gamma}\right\}$. We assume that $\forall s, \boldsymbol{a}^{m, s}=\overline{\boldsymbol{a}}^{m}+$

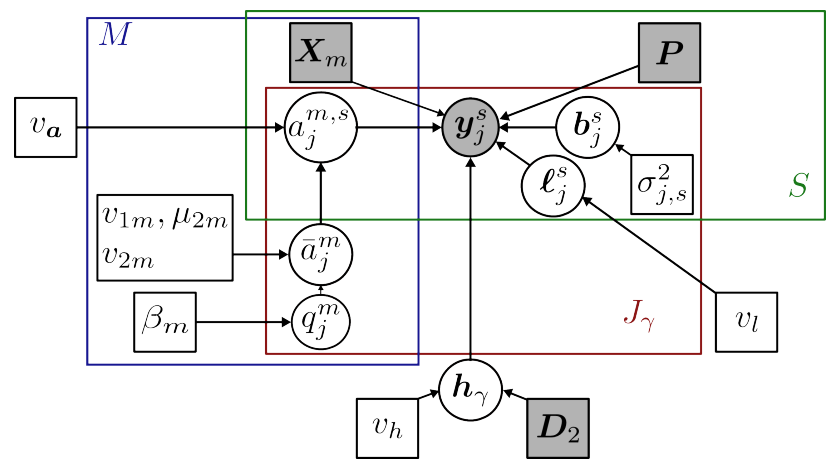

Fig. 1. Directed acyclic graph describing the links between latent and observed variables involved in the MS-JDE approach for a given parcel $\mathcal{P}_{\gamma}$ comprising $J_{\gamma}$ voxels. Circles and squares indicate random variables and model parameters, respectively. Observed variables and fixed parameters are shaded. Plates represent multiple similar nodes with their number given in the plate.

$\boldsymbol{\epsilon}^{s}$ where $\boldsymbol{\epsilon}^{s}$ are independent and identically distributed: $\boldsymbol{\epsilon}^{s} \sim$ $\mathcal{N}\left(\mathbf{0}, v_{\boldsymbol{a}} \boldsymbol{I}_{J_{\gamma}}\right), \forall s$. Notice that for the sake of simplicity, variance $v_{\boldsymbol{a}}>0$ is supposed constant in space and over conditions, what could not be systematically fulfilled in complex paradigms.

Suppose now that the average NRLs $\bar{A}$ act as $\boldsymbol{A}$ in the single session context. Following [1], the variables are supposed independent across experimental conditions: $p\left(\overline{\boldsymbol{A}} \mid \boldsymbol{\theta}_{\overline{\boldsymbol{A}}}\right)=\prod_{m} p\left(\overline{\boldsymbol{a}}^{m} \mid \boldsymbol{\theta}_{m}\right)$ and a spatially adaptive mixture model is introduced on every $\overline{\boldsymbol{a}}^{m}$ through a hidden Markov model on allocation variables $p\left(\boldsymbol{q}^{m} \mid \beta_{m}\right)$, in order to segregate non-activated voxels from activated ones. Hence, the prior $p\left(\overline{\boldsymbol{a}}^{m} \mid \theta_{m}\right)$ reads:

$$
p\left(\overline{\boldsymbol{a}}^{m} \mid \boldsymbol{\theta}_{m}\right)=\sum_{\boldsymbol{q}^{m}}\left[\prod_{j=1}^{J_{\gamma}} p\left(\bar{a}_{j}^{m} \mid q_{j}^{m}, \boldsymbol{\theta}_{m}\right)\right] p\left(\boldsymbol{q}^{m} \mid \beta_{m}\right)
$$

with $\left(\bar{a}_{j}^{m} \mid q_{j}^{m}=i, \boldsymbol{\theta}_{m}\right) \sim \mathcal{N}\left(\mu_{i m}, v_{i m}\right)$ the Gaussian densities associated with the mixture on $\overline{\boldsymbol{a}}^{m}$ and $\boldsymbol{\theta}_{m}=\left\{v_{1 m}, \mu_{2 m}, v_{2 m}, \beta_{m}\right\}$. For non-activating voxels $(i=1)$, we actually set $\mu_{1 m}=0, \forall m$. Finally, we get for the NRLs:

$$
p\left(\mathbb{A}, \overline{\boldsymbol{A}} \mid \boldsymbol{\theta}_{\overline{\boldsymbol{A}}}, v_{a}\right)=\prod_{s} p\left(\boldsymbol{A}^{s} \mid \overline{\boldsymbol{A}}, v_{\boldsymbol{a}}\right) p\left(\overline{\boldsymbol{A}} \mid \boldsymbol{\theta}_{\overline{\boldsymbol{A}}}\right)
$$

with $\left(\boldsymbol{A}^{s} \mid \overline{\boldsymbol{A}}, v_{\boldsymbol{a}}\right) \sim \mathcal{N}\left(\mathbf{0}, v_{a} \boldsymbol{I}_{M \times J_{\gamma}}\right)$.

The other variables $\left(\boldsymbol{H}_{\gamma}, \mathbb{L}, \boldsymbol{\Sigma}\right)$ of the multi-session model have the same priors as those involved in the single session one. Note however that $p\left(\mathbb{L} \mid v_{\ell}\right)=\prod_{s} p\left(\boldsymbol{L}^{s} \mid v_{\ell}\right)$ with $\left(\boldsymbol{L}^{s} \mid v_{\boldsymbol{\ell}}\right) \sim$ $\mathcal{N}\left(\mathbf{0}, v_{\boldsymbol{\ell}} \boldsymbol{I}_{O \times J_{\gamma}}\right)$ and $\left.v_{\boldsymbol{\ell}}\right)>0$. We refer the reader to [1] for details but illustrate on Fig. 1 the dependencies between latent and observed variables involved in the MS-JDE extension.

\section{BAYESIAN INFERENCE}

Let us denote $\Theta$ the set of all hyperparameters related to our variables $\mathbb{A}, \overline{\boldsymbol{A}} \boldsymbol{H}_{\gamma}, \mathbb{L}$ and the noise. Bayesian inference is thus based upon the following joint posterior distribution:

$$
\begin{aligned}
p\left(\mathbb{A}, \overline{\boldsymbol{A}}, \boldsymbol{H}_{\gamma}, \mathbb{L}, \boldsymbol{\Theta} \mid \mathbb{Y}\right) & =p\left(\mathbb{Y} \mid \mathbb{A}, \boldsymbol{H}_{\gamma}, \mathbb{L}, \boldsymbol{\Sigma}\right) \\
& p\left(\mathbb{A}, \overline{\boldsymbol{A}} \mid v_{\boldsymbol{a}}, \boldsymbol{\theta}_{\overline{\boldsymbol{A}}}\right) p\left(\boldsymbol{H}_{\gamma} \mid v_{\boldsymbol{h}}\right) p\left(\mathbb{L} \mid v_{\boldsymbol{\ell}}\right) \\
& {\left[\prod_{\boldsymbol{s}} p\left(\boldsymbol{\sigma}^{s}\right)\right] p\left(\boldsymbol{\theta}_{\overline{\boldsymbol{A}}}\right) p\left(v_{\boldsymbol{h}}\right) p\left(v_{\boldsymbol{\ell}}\right) p\left(v_{\boldsymbol{a}}\right) }
\end{aligned}
$$

Each variable $x$ is sampled using a hybrid Metropolis-Gibbs 
sampling scheme and posterior mean (PM) estimates are computed from the samples according to: $\widehat{x}^{\mathrm{PM}}=\sum_{k=L_{0}}^{L_{1}} x^{(k)} / L, \quad \forall x \in$ $\left\{\mathbb{A}, \overline{\boldsymbol{A}}, \boldsymbol{H}_{\gamma}, \mathbb{L}, \boldsymbol{\Theta}\right\}$ where $L=L_{1}-L_{0}+1$ and $L_{0}$ stands for the length of the burn-in period (see [1] for details). Note that this estimation process has to be repeated over each parcel of the brain.

The session-dependent NRLs follow a posterior gaussian distribution. For the posterior distributions of other variables and the hyperparameters, the reader can refer to [1].

\section{RESULTS}

\subsection{Artificial data}

The following validation on artificial data aims at evaluating the gain in robustness of the multi-session JDE model compared to the single-session formulation. Four sessions data were generated with a varying amount of noise. The first two sessions have a low SNR comparable to the usual SNR encountered in real data and the other two reprsent bad quality data with a very high noise variance. These over-degraded sessions correspond to an extreme case where the data acquisition went wrong. The 4D-BOLD artificial signals were simulated according the observation model in Eq. (2) where $\boldsymbol{h}_{\gamma}$ was set to its canonical shape, $\boldsymbol{P}$ was a polynomial basis of order 4 and $\boldsymbol{X}^{m, s}$ encodes a fast event-related paradigm comprising two conditions (ISI=3.5 s.). For any session $s$, drift coefficients were drawn as $\ell_{j}^{s} \sim \mathcal{N}(0,3.2)$. Noise realisations follow $\boldsymbol{b}_{j}^{s} \sim \mathcal{N}\left(0, \sigma_{,, s}^{2}\right)$ with $\sigma_{., 1}^{2}=\sigma_{., 2}^{2}=.6$ (low SNR) and $\sigma_{., 3}^{2}=\sigma_{,, 4}^{2}=16$ (very low SNR). For any session, neural response levels were drawn according to: $\left(a_{j}^{m, s} \mid q_{j}^{m}=2\right) \sim \mathcal{N}(2.5,0.3)$ (activating class) and $\left(a_{j}^{m, s} \mid q_{j}^{m}=1\right) \sim \mathcal{N}(0,0.3)$ (inactivating class). Finally, as shown on the top-right map of Fig. 2 for $m=1$, activation states (labels $\boldsymbol{Q})$ were set by hand-drawn maps. The single-session JDE model (SS-JDE) was applied to each session independently and the multisession version (MS-JDE), to all sessions jointly. For the sake of simplicity, results are shown only for the condition $m=1$ (the same conclusions hold for $m=2$ ).

Fig. 2 shows results for the neural response levels and labels. The first two columns correspond to the "normal" low SNR situation: the estimated NRL maps are comparable to the true maps for all JDE versions. The next two columns show the highly noisedegraded sessions: the SS-JDE NRL maps are much less sensitive than the ones obtained by MS-JDE, which is able to recover results closer to the ground truth. For a fairer comparison, the mean of the SS-JDE NRL maps were computed and compared to the NRL average maps estimated by MS-JDE (see the fifth column of Fig. 2, second and third lines). We observe that the estimated mean response levels of MS-JDE is much closer to the average true values than the NRLs obtained by the SS-JDE. We can thus conclude that the MSJDE model enables to regularize response levels across sessions and adapts to the actual amount of noise within sessions. The same conclusions can be formulated based upon results shown on the average of in the rightmost column of Fig. 2: the label maps estimated by SSJDE are compared to the label map governing all sessions obtained by MS-JDE.

The HRF estimation is less impacted by the noise-degraded sessions for the SS-JDE model, as depicted Fig. 3. More precisely, on Fig. 3(a), HRFs estimated for the more degraded simulated sessionsdata (in red and cyan) are deformed compared to the true shape. The peak and undershoot timings are shifted of $\sim 1.5 \mathrm{~s}$ and we observe a larger peak width. In contrast, HRFs estimated for the normal sessions-data (in green and blue) are closer to the true one (black
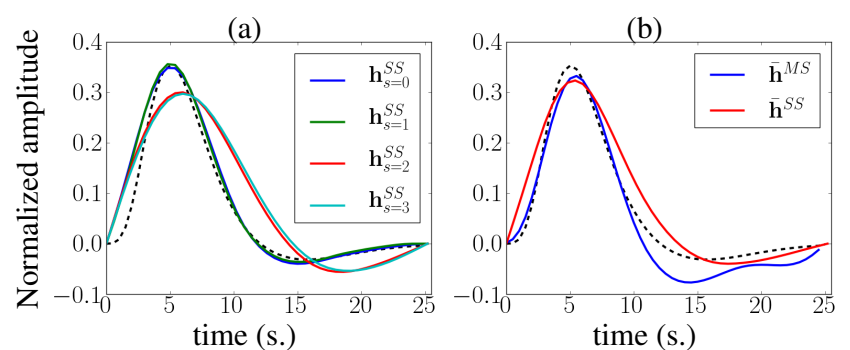

Fig. 3. HRF estimation results on artificial data. (a): HRF estimates for each session obtained with the SS-JDE model $(S S)$. (b): Mean of HRF estimates over sessions obtained by SS-JDE (red) and HRF estimate obtained by MS-JDE (blue). For both plots, the ground truth in black dashed line fixed to the same shape for all sessions/runs.

dashed curve). However, the average of HRFs estimated by SS-JDE on Fig. 3(b) (in red) remains comparable to the true simulated HRF. Besides, the HRF estimated by the MS-JDE model is close to the true shape around the peak, but departs more from it around the undershoot. This can be explained by the fact that the HRF tail is more difficult to estimate in this part, since the signal strength is low.

\subsection{Experimental results}

Real fMRI data were recorded during an experiment designed to map auditive and visual brain functions, which consisted of a six sessions of $N=125$ scans lasting $T R=2.4 \mathrm{~s}$, each yielding a 3 -D volume composed of $64 \times 64 \times 32$ voxels. The paradigm was fast event-related comprising sixty auditive and visual stimuli.A region of interest in the right temporal lobe was defined manually and parcellated into 10 functional parcels. The MS-JDE and SS-JDE methods were compared using the same process as for the analysis of articial data, but only results on global means are shown.

As depicted in Fig. 4, the MS-JDE model provides more sensitive NRLs maps than the SS-JDE version. Indeed, the NRL means estimated by MS-JDE jointly over all sessions (Fig. 4(b)) are more contrasted and have a higher peak value than the estimates obtained independently by SS-JDE and averaged over sessions (Fig. 4(a)). In fact, averaging the session-specific SS-JDE results lowers the overall response levels due to the inter-session variability. In contrast, estimating the overall response mean while spatially regularizing as done by MS-JDE enables to enhance the response level recovery. Besides, the inter-session variability is illustrated in the HRF estimates obtained by SS-JDE in Fig. 5(a). As observed for the artificial data results, the HRF overaged over sessions for SS-JDE and the MS-JDE HRF estimate are quite close.

\section{CONCLUSION}

In this paper, we extended the previous JDE model [1] to jointly process different sessions recorded for a given subject. Results from artificial and real data showed that the new hierarchical model is well adapted to robustly recover activated areas from multi-sessions data with a slight improvement in the HRF estimation, which is particularly useful when some sessions are of poor quality and associated with a very low SNR. To justify the practical value of the proposed method, further validation on real fMRI dataset are currently performed. Thus, we plan to benefit from the advantages of the JDE framework in cognitive studies focusing on cerebral specialization in 


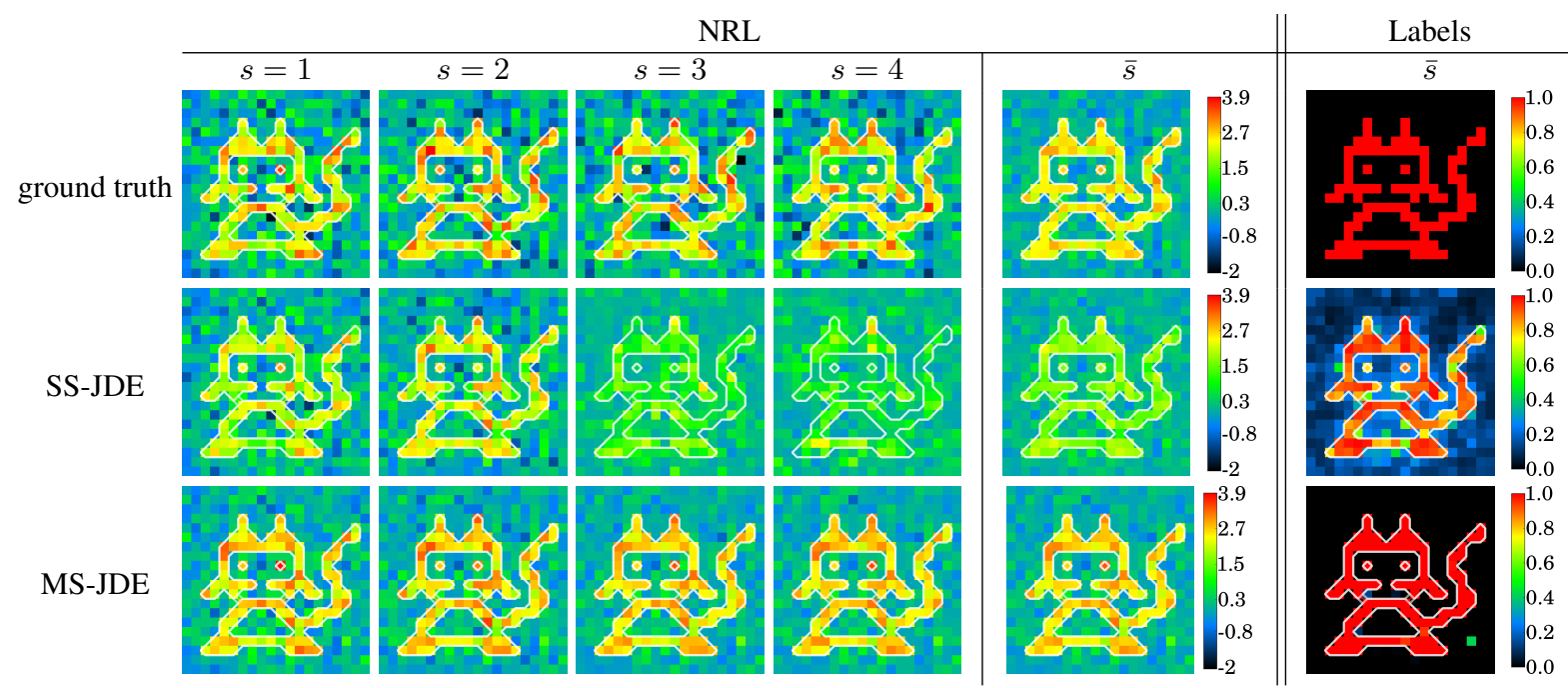

Fig. 2. Top row: True session-varying NRLs and labels $(s=1$ to $s=4)$ used for simulating artificial datasets. Middle row: Sessiondependent NRL and label estimates infered by the SS-JDE approach. Bottom row: Session-dependent NRL and label estimates infered by the MS-JDE extension. The fifth column (NRL, $\bar{s}$ ) provides the mean NRL values for the ground truth (top), the SS-JDE inference scheme (middle) and the estimates of NRL means obtained by the MS-JDE extension (bottom). The sixth column (Labels, $\bar{s}$ ) provides the label values for the ground truth (top), the mean of label estimates for the SS-JDE inference scheme (middle) and the label map obtained by the MS-JDE extension (bottom).

(a)
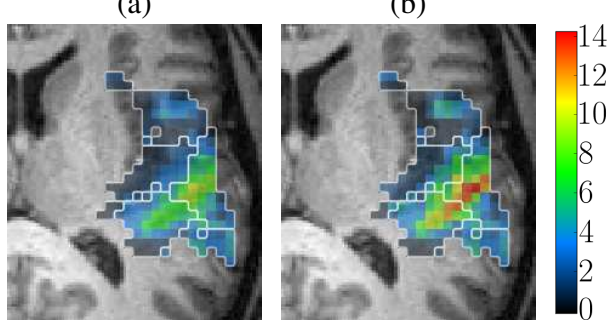

Fig. 4. NRL estimation results on real data (zoomed on a right temporal region). (a): Mean of NRL estimates over sessions obtained with the SS-JDE model. (b): Estimates of NRL means obtained with MS-JDE jointly on all sessions.
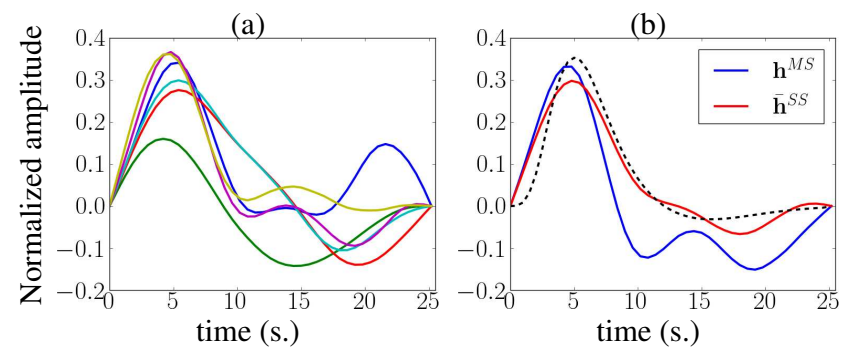

Fig. 5. HRF estimation results on real data. (a): HRF estimates for each session obtained with the SS-JDE model. (b): Mean of HRF estimates over sessions obtained by SS-JDE (red) and HRF estimate obtained by MS-JDE (blue). The canonical HRF is depicted as a black dashed line. infants linguistic processing. Indeed, multi-session data are usually required in this context, mostly because of the difficulty of acquiring long datasets in the young age. For such studies, the MS-JDE model enables an extensive analysis of the hemodynamic variability along the Superior Temporal Sulcus.

\section{REFERENCES}

[1] T. Vincent, L. Risser, and P. Ciuciu, "Spatially adaptive mixture modeling for analysis of within-subject fMRI time series," IEEE Trans. Med. Imag., vol. 29, no. 4, pp. 1059-1074, Apr. 2010.

[2] L. Chaari, F. Forbes, T. Vincent, M. Dojat, and P. Ciuciu, "Variational solution to the joint detection estimation of brain activity in fMRI," in 14th Proc. MICCAI, Toronto, Canada, Sep. 2011, LNCS 6892 (Part II), pp. 260-268, Springer Verlag Berlin Heidelberg.

[3] S. Badillo, T. Vincent, and P. Ciuciu, "Impact of the joint detectionestimation approach on random effects group studies in fMRI," in 8th Proc. IEEE ISBI, Chicago, IL, Apr. 2011, pp. 376-380.

[4] D. A. Handwerker, J.M. Ollinger, and M. D'Esposito, "Variation of BOLD hemodynamic responses across subjects and regions and their effects on statistical analyses," Neuroimage, vol. 21, pp. 1639-, 2004.

[5] B. B. Zandbelt, T. E. Gladwin, M. Raemaekers, M. van Buuren, S.F. Neggers, R.S. Kahn, N.F. Ramsey, and M. Vink, "Within-subject variation in BOLD-fMRI signal changes across repeated measurements: quantification and implications for sample size," Neuroimage, vol. 42, no. $1,2008$.

[6] S.M. Smith, C.F. Beckmann, N. Ramnani, M.W. Woolrich, P.R. Bannister, M. Jenkinson, P.M. Matthews, and D.J. McGonigle, "Variability in fMRI: a re-examination of inter-session differences." Hum. Brain Mapp., vol. 24, no. 3, pp. 248-, 2005.

[7] B. Thirion, G. Flandin, P. Pinel, A. Roche, P. Ciuciu, and J.-B. Poline, "Dealing with the shortcomings of spatial normalization: Multi-subject parcellation of fMRI datasets," Hum. Brain Mapp., vol. 27, no. 8, pp. 678-, 2006.

[8] J. Neumann and G. Lohmann, "Bayesian second-level analysis of functional magnetic resonance images," Neuroimage, vol. 20, no. 2, pp. 1346-, 2003. 